\title{
DEBATES
}

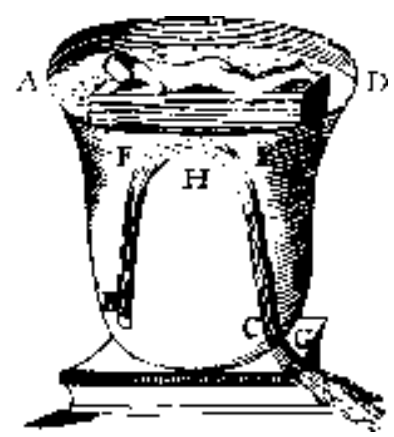

La sección Debates presenta en este número un artículo que analiza críticamente una de las nuevas asignaturas del Bachillerato: Ciencias de la Tierra y del Medio Ambiente. Una materia que se presenta algo indefinida en el panorama de una reforma que no da sensación de una gran solidez estructural.

En estos momentos se advierte un cierto desconcierto en el colectivo de profesores de los centros en los que se va implantando esta materia, fruto sin duda de la ambigüedad que se refleja ya en su propio título.

Por ello, pensamos que este artículo es oportuno, ya que aporta argumentos para la realización de un análisis serio de la razón de ser de una asignatura llamada Ciencias de la Tierra y del Medio Ambiente en el nuevo Bachillerato.

\section{POR UNAS CIENCIAS AMBIENTALES Y UNAS CIENCIAS DE LA TIERRA. REFLEXIONES CRÍTICAS Y PROPUESTAS PARA UN DEBATE}

PASCUAL TRILLO, J.A.

Centro de Profesores y de Recursos Madrid-Norte. Pza. La Remonta, s/n. 28039 Madrid.

\section{SUMMARY}

Earth and Environmental Sciences are analysed in criticism form. A differentiation of Environmental Sciences and Earth Sciences as different subjects is proposed. In the case of Environmental Sciences, a «taxonomy» of contents is included with the purpose of facilitating the programme of the subject. Environmental Sciences should be a trunk subject for all the students in PostObligatory Secondary Education («bachillerato»), while Earth Sciences, a new suggested subject, should be a subject only for the modality of Sciences. 


\section{LAS CIENCIAS DE LA TIERRA Y DEL ME- DIO AMBIENTE}

Las Ciencias de la Tierra y del Medio Ambiente (a partir de ahora CTMA) se han configurado como una materia propia de la modalidad del bachillerato denominada Ciencias de la Naturaleza y de la Salud (RD 1700/1991), ubicándose en el segundo curso de la modalidad de Ciencias de la Naturaleza y de la Salud (RD 1179/1992). Esta incorporación en la educación secundaria de una materia específicamente dirigida al tratamiento del medio ambiente ha discurrido de forma más o menos paralela a la aparición de una licenciatura de Ciencias Ambientales en los estudios universitarios (RD 2083/1994). En el caso de la enseñanza secundaria, la aprobación vino precedida de una importante polémica relacionada con el «desplazamiento» de la geología de las materias propias, quedando sólo como optativa (AEPECT, 1992). La CTMA se adscribió al «cuadro» de las disciplinas científicas y fue asignada al Departamento de Biología y Geología.

No es fácil delimitar una materia tan compleja y «transdisciplinar» como son las «ciencias ambientales» en ningún ámbito o nivel educativo. En el espacio universitario hay ejemplos sobrados de las dificultades habidas, que en algunos casos han acabado por imposibilitar la configuración de los estudios en algunas universidades. Allí da la impresión de que el reparto de las asignaturas entre los departamentos constituye el verdadero caballo de batalla para la configuración de los estudios y las titulaciones. En el caso del bachillerato, la llegada de las ciencias del medio ambiente tampoco ha estado exenta de polémicas. El que algunas personas pusieran en este empeño mucho de su tiempo y no pocos esfuerzos representa una labor que a ellos les honra y a nosotros nos permite estar hoy aquí discutiendo acerca de los posibles puntos flacos y de las posibilidades de mejora. Vaya, pues, por adelantado, nuestro reconocimiento a su tarea. Pero creemos que ha llegado el tiempo de reflexionar sobre lo que tenemos y aportar algunos planteamientos críticos a la materia tal como se ha configurado, con el fin de contribuir en alguna medida a abrir un debate orientado a su posible mejora, algo que nos parece no sólo posible, sino francamente necesario. Pero no se nos malinterprete: partimos de la creencia de que la materia supone un «paso cualitativo» adelante en la integración de la comprensión y el análisis de lo ambiental en la enseñanza secundaria $y$, por lo tanto, es un peldaño necesitado de fuertes reparaciones, pero indispensable.

\section{LAS CIENCIAS DE LA TIERRA}

El primer aspecto que llama la atención en la materia es la conjunción de dos «disciplinas» en el título de la misma. La unión de las ciencias de la Tierra y de las ciencias del medio ambiente requiere, cuando menos, una primera reflexión.

Si quisiéramos buscar una referencia epistemológica para las ciencias de la Tierra, encontraríamos no una, sino varias. La primera, y posiblemente la más asentada entre nosotros, es la que entiende las ciencias de la Tierra como un nuevo campo de estudio, interpretación y comprensión de la Tierra física como un sistema planetario complejo y global. Nacería esta concepción de una «evolución» conceptual de la geología que, para algunos, se habría quedado «reducida», anquilosada e incapaz de progresar como disciplina «suficiente» desde que la formulación de la tectónica global y el desarrollo de nuevos campos con herramientas conceptuales y tecnológicas propias, como la planetología, la meteorología, la geofísica u otras, habría cambiado el «núcleo duro» de los programas de investigación sobre el planeta Tierra. Por tanto, estaríamos ante un cambio de paradigma, por utilizar la conocida expresión de Thomas Kuhn (1962), o frente a un nuevo programa de investigación, de preferir a Imre Lakatos.

Entre los valedores de este salto cualitativo de la geología a las ciencias de la Tierra destaca Tuzo Wilson, quien sostenía ya en 1968 que «el campo de la geología se ha reducido» (Wilson, 1968), y advertía que los expertos en temas -que para él eran clave- como los cambios relativos del límite entre tierra y mar, los terremotos o el calor terrestre «son los geodestas y los geofísicos, y pocos de ellos han recibido una formación sólida en geología». Sostenía, Wilson, que «parece que hay que volver a examinar en su conjunto el programa de las ciencias de la Tierra y que asistimos a un cambio semejante al que se produjo cuando la física moderna reemplazó la física clásica en la enseñanza universitaria. Debemos estudiar la Tierra como un todo, como un sistema único». Esta visión trataba de integrar el incipiente enfoque de sistemas a la Tierra como un todo geofísico, buscando hallar solución a lo él definió como «la pérdida de prestigio de la geología clásica». De entre todas las «versiones» posibles sobre lo que representarían las ciencias de la Tierra, ésta es, seguramente, la que más valedores encuentra entre los científicos de la naturaleza de nuestro país, especialmente en el ámbito de la geología o de la física. Desde esta perspectiva, el «núcleo duro» correspondería a la tectónica de placas, una auténtica «teoría de la Tierra» como ha sido definida (Anguita, 1996).

Esta evolución se manifiesta, por ejemplo, en la transformación de la antigua Association of Teachers of Geology británica a la actual Earth Science Teachers's Association, un proceso que tiene también reflejo en España en la creación de la activa Asociación Española para la Enseñanza de las Ciencias de la Tierra (AEPECT). En ambos casos, la línea orientadora de las ciencias de la Tierra estriba en la convergencia de la geología con la geofísica, la geoquímica, la oceanografía, la climatología y la meteorología (Anguita, 1994). Sin embargo, también hay que advertir que en el caso británico, que ha sido uno de los países con mayor movimiento didáctico orientado hacia la idea de las ciencias de la Tierra, la concreción de éstas en los currículos de secundaria no ha conseguido superar la de 
unas «ciencias naturales» (biología más geología), del tipo de las tradicionalmente existentes en los currículos españoles (King, 1992).

Sin embargo, no es éste el único planteamiento posible para las ciencias de la Tierra. Así, la geografía física, tradicional, campo limítrofe de la geografía con la geodinámica y la geomorfología, mantiene un constante interés por la explicación global de la superficie del planeta Tierra y los paisajes mundiales en una posible acepción de ciencias de la Tierra: encontraremos este tipo de enfoque en la propuesta de una «ciencia del paisaje» en geógrafos (Bolós, 1992) o en ecólogos (González Bernáldez, 1981), así como en la denominada ecogeografía propuesta por los franceses Jean Tricart y Jean Killian (1979), procedentes, respectivamente, de los campos de la hidrogeografía y la edafología. En este último caso se busca la confluencia entre lo ecológico y lo geográfico, como comprensión espacial de los sistemas ecogeográficos, buscando la aplicación de modelos de ordenación territorial y planificación física (ésta suele ser la principal forma de incorporación de los departamentos de geografía a las nacientes licenciaturas españolas en ciencias ambientales). Contenidos tradicionales de estas visiones ambientales de la geografía y la geodinámica son los relacionados con el territorio y su ordenación, los paisajes o los riesgos geológicos y climáticos (que sí aparecen entre los contenidos de CTMA), particularmente por su relación con la planificación territorial. Esta segunda aproximación, menos consolidada aquí bajo la denominación de ciencias de la Tierra, estaría más cercana, pues, a la idea de lo que se va configurando como el campo emergente de las ciencias ambientales.

Acepciones muy diferentes de otras ciencias de la Tierra son aquéllas en las que el núcleo de interés se orienta hacia la historia de la vida como el aspecto particular y definitivamente personalizador de nuestro planeta. Aunque cabría esperar que este tipo de propuestas viniera del campo de la biología (y, en parte es así), también hay geólogos, paleontólogos y químicos entre los principales implicados en la creación de esta línea epistémica. Si hubiera que reducir a dos los nombres de los principales responsables de la misma, nos quedaríamos con los de la bióloga de Boston y Massachusetts, Lynn Margulis, una auténtica revolucionaria del campo de la biología evolutiva, y con el químico independiente James Lovelock, el principal impulsor de la controvertida teoría Gaia. Tal vez el centro irradiante de esta visión, que cabe también tildar de ciencias de la Tierra («viva»), sea el de los cursos de evolución ambiental (Margulis y Olendzenski, 1992), herederos de las propuestas del ruso Valdimir I. Vernadsky y apoyados por las investigaciones de la oficina de las Ciencias de la Vida de la NASA. Esta nueva biología holística o sistémica ha recibido también la denominación, algo enrevesada, de «biogeoquímica evolutiva planetaria».

Varios son, pues, los antecedentes existentes sobre los que sustentar el término de «ciencias de la Tierra», desde la geofísica a la biogeoquímica, con o sin inclusión de la vida como explicación u objetivo central de nuestros intereses (desde la Tierra como sistema geofísico a la Tierra como Gaia). La pregunta que cabe formularse es: ¿De cuál se reclaman deudoras las ciencias de la Tierra de la materia de bachillerato?

En el currículo oficial, sin embargo, no hemos encontrado una respuesta clara a esa pregunta. Habríamos apostado por la primera interpretación, sobre todo debido a las causas que se adivinan en la conversión de las ciencias medioambientales de la primera propuesta ministerial a las actuales ciencias de la Tierra y del medio ambiente (confrontación con la geología y, tal vez, con la geografía); pero llama la atención la ausencia explícita del «núcleo duro» de tales ciencias de la Tierra entre los contenidos oficiales: no aparece la tectónica de placas de forma explícita, aunque se pueda adivinar tras el apartado de geosfera que incluye, de todos modos, también la tradicional tríada de procesos geodinámicos externos (erosión, transporte y sedimentación). Tampoco es suficiente el peso de la oceanografía física, la meteorología o la geofísica en el currículo como para justificar una ciencias de la Tierra «wilsonianas».

Pero menos encajan las otras interpretaciones (particularmente las últimas), dado que no hay alusión ni directa ni indirecta al origen y evolución de la vida en la Tierra, si no es en la forma reducida incluida en el epígrafe «La humanidad y el medio ambiente» como «los cambios ambientales en la historia de la Tierra como resultado de las interacciones entre la atmósfera, la hidrosfera, la biosfera y la geosfera» (que, además cabe interpretar más como una propuesta de introducción al análisis de las relaciones entre la humanidad y el medio ambiente, ya que aparece bajo ese título general). Desde luego, mucho más próximo al concepto wilsoniano de ciencias de la Tierra encontramos los currículos de «Biología y geología» de $1^{\circ}$ y de «Geología» de $2^{\circ}$, que no recogen, sin embargo, este término.

En la introducción del currículo se explica que se trata de una propuesta para el estudio de los sistemas terrestres. Por tanto, la concreción en los contenidos estriba en los que están englobados bajo este epígrafe («geosfera», «atmósfera e hidrosfera», «biosfera» e «interfases entre las capas terrestres»). Sin embargo, esta parte constituye, a nuestro juicio, un bloque poco integrado con el resto de la materia, provocando con ello no pocos problemas para conseguir un tratamiento coherente de toda ella: en el fondo, casi consiste en «embuchar» todos los contenidos de geología, geomorfología, ecología, geofísica de las capas fluidas, climatología, etc. (aunque muy parcialmente definidos) en el interior de una materia que se dirige mayoritariamente a otros fines. Su «aislamiento» del resto es una consecuencia natural de lo anterior. Como trataremos de demostrar, la «incorporación» que se pretende de la parte de «ciencias de la Tierra» a la materia no supone una aportación integradora y positiva, sino que introduce importantes dosis de confusión y de abstracción, y abre posibles vías por las que algunos han llegado a considerar que una pregunta del tenor de «diferencias entre granitos y calizas» puede representar una opción razonable para una prueba de acceso a la universidad de esta materia. 
En resumen, no vemos en la incorporación de las ciencias de la Tierra al título de la materia, sino un elemento más perturbador que positivo; particularmente si aquélla parte, como todo apunta, de una concepción de «nueva geología». De ser así, también valdría un título como «Geofísica y Ciencias del Medio Ambiente»; y, en ese caso, ¿por qué no crear también una «Ecología y Ciencias Ambientales» o una «Bioquímica y Ciencias Ambientales»? Y así hasta el absurdo.

Si lo que se pretendía en realidad (y apoyamos fervientemente) era crear un espacio en el bachillerato a las «ciencias de la Tierra», entendidas de la forma que antes se expresó (geología + climatología + meteorología + oceanografía física + geofísica + geoquímica), pónganse los medios para hacerlo bien y sensatamente, pero no se alcancen medias tintas que nada bueno deparan, pretendiendo dar un medio gusto a todos y no conformar a ninguno (un «medio» ambiente que se hace «cuarto y mitad» y unas ciencias de la Tierra que se quedan en mero repaso de algunos temas de ecología y geología). Las ciencias de la Tierra deberían encontrar una ubicación digna (y no por la puerta trasera) en el último curso del bachillerato científico. Es más: creemos que debieran ser lo más «globales» posible, integrando tanto los nuevos territorios científicos de la «teoría de Gea» (si así podemos llamar al paradigma de las ciencias «wilsoninanas» sobre la Tierra física), como los de la teoría de Gaia de Margulis-Lovelock. Se generaría así un espacio educativo en bachillerato sumamente interesante y actual, capaz de permitir en esta etapa educativa una aproximación inicial a algunos de los más interesantes aspectos científicos sobre la comprensión de nuestro planeta y de la vida.

Aunque aquí no entraremos a especular sobre las características que habría de tener tal materia, sí apostamos decididamente por ella: defendemos el establecimiento de una materia de esas características con el rango y dignidad que requiere, pero separada, decididamente, de las ciencias ambientales.

\section{LAS CIENCIAS AMBIENTALES}

La mayor parte de los objetivos y contenidos de la materia de bachillerato se refiere, de todos modos, al amplio campo de las llamadas «ciencias ambientales», un campo del conocimiento «en construcción» y constante evolución, que requerirá, sin duda, de constantes actualizaciones. Tal vez por la necesidad de «hacer hueco» a lo que pudieran representar «las ciencias de la Tierra» de las que antes hemos hablado, o por otras razones, lo cierto es que se detectan ausencias relevantes (por ejemplo: ¿qué hay de la demografía o de la ecología humana, fundamentales en el análisis ambiental?), así como una cierta desorientación en la exposición de los contenidos, sobre la que luego insistiremos.

Desde luego que con el tiempo y las posibilidades que una materia de bachillerato puede ofrecer a la enseñanza-aprendizaje del medio ambiente y los problemas relacionados, las ciencias ambientales encontrarán siempre serias limitaciones en esta etapa, derivadas tanto de su amplitud y complejidad como de su profundo carácter interdisciplinar (si no transdisciplinar), lo que exige, entre otras cuestiones, una fuerte demanda de actualización en la formación del profesorado si no se quiere que la materia termine siendo una especie de «biología y geología», aunque denominada de otro modo.

Una verdadera aproximación a las ciencias ambientales en el bachillerato supone una materia orientada al estudio, comprensión, análisis e interpretación del medio ambiente y de los problemas ambientales generados como consecuencia de las actuaciones humanas sobre el sistema físico-natural, contemplando en ella las perspectivas ecológica, económica, social, política, ética y tecnológica (una idea de la globalidad de los contenidos de las ciencias ambientales puede obtenerse consultando el índice de libros escritos y utilizados para cursos de esta disciplina, entre los que puede destacarse el de Miller, 1992). Las principales bases necesarias para este enfoque están afortunadamente incluidas entre los objetivos y los contenidos del currículo oficial y podría decirse que en ellas estriba la orientación y justificación de la CTMA. Pero algunos aspectos (entre los que se incluye la cuestión de las ciencias de la Tierra y otros sobre los que volveremos más adelante) pueden desvirtuar en la práctica tal posibilidad.

\section{Los objetivos}

La definición de los objetivos de la materia es, a primera vista, interesante. Es fácil advertir una ordenación interna de los objetivos en función de la búsqueda de capacidades de tipo conceptual-factual, procedimental y axiológico, conforme a la conocida clasificación de contenidos.

Puede advertirse que, desde las capacidades de tipo cognitivo, se hace hincapié en comprender cuestiones de funcionamiento, explicar repercusiones, analizar causas, conocer medidas y límites. Se establecen, pues, objetivos graduados de conocimiento en función de los contenidos a los que se orientan tales capacidades. Además, el mayor grado de aprehensión conceptual de entre los que aparecen («poder explicar») se orienta a establecer una relación entre los hechos locales y las repercusiones mundiales. En este sentido, cabría interpretar que los objetivos más ambiciosos se sitúan en la línea de los contenidos conceptuales que podríamos considerar más propios de las ciencias ambientales (por ejemplo, la relación entre lo local y lo global en el medio ambiente). El resto de los objetivos de este tipo, a excepción del relativo a los recursos, se dirige hacia aspectos que la propia «introducción» adscribe a la parte de las ciencias de la Tierra (los sistemas terrestres) y a los riesgos naturales (que incluyen los de tipo geológico y climático, pero ignoran otros tipos, incluidos los de tipo biológico o sanitarios, como las plagas, epidemias, etc.). Finalmente, se hace una alusión directa a los límites en la explotación de los recursos, destacando este aspecto concreto de la problemática ambiental que tanto preocupó al Club de Roma en los años setenta. Sin embargo, no 
hay indicación explícita de la problemática relativa a las otras dos fuentes de males ambientales, hoy consideradas aún más graves para el ambiente mundial que la cuestión de los límites de los recursos: los residuos (outputs o «salidas», en la jerga económico-ecológica) y la ocupación/alteración de los sistemas ecológicos. Sin duda, es posible considerarlos desde la aludida relación entre lo local y lo global, lo que también valdría para el caso de los recursos o de los riesgos, aunque éstos sí se mencionan explícitamente.

Los objetivos relativos a las capacidades del «saber hacer» se dirigen a evaluar rentabilidades, investigar problemas y utilizar técnicas. Sin duda, la capacidad de investigar los problemas ambientales es un aspecto clave que, en realidad, engloba y subordina a las otras dos, por lo que realmente constituye la pauta de los objetivos en cuanto a los procedimientos que hay que aprender. Se trata de un objetivo acertado, aunque, claro está, necesitado de concreción en la práctica, pues en la forma en que se formula es tan válido aquí como al final de la licenciatura de ciencias ambientales o del doctorado.

Finalmente, se incluyen dos objetivos referentes a los valores, que buscan adquirir conciencia y mostrar actitudes. Ciertamente, la redacción del segundo plantea problemas casi irresolubles para su evaluación, pero, como veremos, los criterios de evaluación soslayan francamente la evaluación de ambos objetivos (salvo, tal vez, en el último criterio, que busca que se asegure conocer algunas medidas que posibiliten la aplicación de tales actitudes en comportamientos, lo que desde luego no es poco).

Respecto a los objetivos, se encuentra una orientación válida para las ciencias ambientales, aunque realmente podría hacerse con un mayor esfuerzo de síntesis, de forma que se resumieran en:

- adquirir las capacidades de conocer, comprender y explicar las causas y consecuencias de los principales problemas ambientales y del propio funcionamiento del medio ambiente;

- plantear y realizar pequeñas investigaciones sobre aquéllos capaces de ofrecer explicaciones y dar orientación en las soluciones a los mismos; y

- ser sensibles y activos en la preocupación ambiental desde una perspectiva democrática solidaria y ética con la naturaleza y el resto de la humanidad.

\section{Los contenidos}

De los contenidos, cabe decir que no hay una lógica o coherencia suficiente en la ordenación dada bajo los epígrafes propuestos. Sin duda, se encuentra una aceptable cantidad de contenidos capaz de posibilitar programaciones más que amplias en materia de ciencias ambientales, pero también se introduce una considerable confusión a través de la forma en que se presentan, así como por la existencia de algunos aspectos más que cuestionables. Vayamos por partes.

Aparte del apartado de «Aproximación al trabajo científico», presente en todas las materias científicas del bachillerato, hay cuatro epígrafes a modo de «bloques». Son: «La humanidad y el medio ambiente», «Los sistemas terrestres», «Las relaciones entre la humanidad y la naturaleza» $\mathrm{y}$ «Medio ambiente y desarrollo sostenible». Con excepción de «Los sistemas terrestres», no se advierte claridad en todo ello ni sobre lo que luego engloban, abusando de la reiteración. Si nos asomamos al interior, encontraremos un caos importante. A modo de ejemplo: tras el epígrafe de «La humanidad y el medio ambiente», hallaremos «La teoría de sistemas» o «Los cambios ambientales en la historia de la Tierra como resultado de las interacciones entre la atmósfera, la hidrosfera, la biosfera y la geosfera», mientras que «La evolución de las relaciones entre la humanidad y la naturaleza» no se encuentra, como cabría esperar, en el bloque de «Las relaciones entre la humanidad y la naturaleza», sino en el apartado de «La humanidad y el medio ambiente». Los «Impactos ambientales» se encuentran en el apartado titulado «Las relaciones entre humanidad y naturaleza», pero «La evaluación del impacto ambiental» aparece bajo el epígrafe de «Medio ambiente y desarrollo sostenible». Verdaderamente, si los epígrafes dicen poco e informan menos, podrían haberse eliminado: tal como aparecen causan más confusión que claridad.

Creemos, por tanto, que desprenderse de tales títulos, desmantelar los supuestos «bloques» y empezar a ordenar de otra forma los contenidos, agrupándolos de nuevo, es un ejercicio recomendable para el profesorado.

Un caso aparte, lo constituye el apartado «Sistemas terrestres». Agrupa, como vimos, lo que de «ciencias de la Tierra» tiene la materia, de acuerdo con la «introducción», pero resulta ser un conjunto parcial y poco definido de contenidos sobre energética terrestre, geodinámica externa, climas y dinámica de capas fluidas y ecología A ello se añade un último apartado realmente desconcertante, pues, aunque parece querer enfocar el estudio de las interfases entre los anteriores sistemas terrestres, termina ofreciendo una relación parcial y confusa de ¿ecosistemas?, ¿ecotonos?, sin aclarar su finalidad en el contexto general y sin que haya tampoco referencia posterior en los criterios de evaluación. Por otra parte, la presentación de los contenidos de los sistemas terrestres, lejos de favorecer un tratamiento o enfoque sistémico por el que se dice apostar (y que resulta sumamente adecuado en las ciencias ambientales), conduce más fácilmente a un planteamiento opuesto de tipo reduccionista: geología, biología, climatología... ¿Pero no quedamos en que el objetivo en el estudio del medio ambiente es el propio sistema ambiental? ¿Cómo pretender, pues, un enfoque sistémico si se separa la «energía de la geosfera» de la «energía de los ecosistemas» y de la «energía de la atmósfera e hidrosfera», como parece deducirse de la organización de contenidos expuesta? Aunque este bloque favorece la inclusión de numerosos contenidos, queda inconexo y aislado, y, sobre todo, se convierte en una fuente poten- 
cialmente introductora de enfoques y contenidos difícilmente coincidentes con el tratamiento «ambiental» de la orientación primordial de la materia. Por todo ello, resulta francamente prescindible.

En cuanto a los otros contenidos, más coherentes con el núcleo propio de las ciencias ambientales, contienen también algunos problemas no sólo en cuanto a su presentación y ordenación (ya comentados), sino por la presencia de imprecisiones e, incluso, de «errores». No se ofrece un marco sistémico que englobe los temas tratados (o ayude a hacerlo), lo cual se facilitaría si se presentasen los temas en función de su relación con las «entradas» (inputs) al sistema socioeconómico desde el medio natural (cuestiones relativas a los recursos), con las «salidas» (outputs) de aquél (cuestiones relativas a los residuos, emisiones, efluentes: contaminación en suma), o de los problemas derivados de la ocupación, alteración y generación de tendencias y dinámicas en los ecosistemas (riesgos, degradación y destrucción de sistemas ecológicos, etc.). Tampoco se propicia el análisis de la crisis ambiental y de las repercusiones sociales a una escala global, necesario, por otra parte, para abordar el acierto (ese sí) de incluir la cuestión del desarrollo sostenible como contenido clave de la materia. Sin embargo, en este punto es preciso hacer una referencia especial, debido a su importancia y a las posibles repercusiones, de la inadecuada referencia a dos modelos: «conservacionista» y de «desarrollo sostenible», tanto en los contenidos como (lo que agrava el problema) en los criterios de evaluación. Lo veremos a continuación.

\section{La cuestión de los modelos de desarrollo}

A estas alturas, la consideración de respuestas globales para los problemas ambientales es un contenido indispensable en todo programa de ciencias ambientales. Ello lleva siempre a la necesidad de tratar la causa última de aquéllos: los modelos de desarrollo. La búsqueda de una forma alternativa de compatibilizar la conservación del medio ambiente con el desarrollo humano, denominada desde el informe de la Comisión Brundtland (WCED, 1987), como «desarrollo sostenible», y posteriormente avalada, completada y reforzada desde otros foros internacionales, entre los que destaca la Cumbre de la Tierra y su Agenda 21 (oficialmente traducido aquí como Programa 21), constituye un tema indudablemente clave dentro de la temática ambiental actual e, incluso, en el ámbito de la progresiva evolución de la educación y de la enseñanza de las ciencias en particular, como algunos han propuesto (Sáez y Riquarts, 1996).

El desarrollo sostenible entra, pues, en el currículo del bachillerato a través de las ciencias de la Tierra y del medio ambiente, lo que representa un indudable acierto. Sin embargo, no lo es la forma en que se presenta: enfrentado a un supuesto «modelo conservacionista», tanto en los contenidos del currículo oficial como en los criterios de evaluación (criterio 10: «Diferenciar ante un problema ambiental los argumentos del modelo "conservacionista" y los del "desarrollo sostenible"»). Porque... ¿cuál es el «modelo conservacionista»?
Si hubiera que rastrear la pista a ese supuesto modelo, lo más lógico sería suponer que se trata del modelo propuesto por el sector conservacionista, es decir, aquéllos que han venido promoviendo la conservación de la naturaleza. Afortunadamente se trata de un sector identificable a escala mundial, aunque muy amplio. El referente más global sería el representado por la UICN Unión Mundial para la Naturaleza, la organización internacional que agrupa estados, agencias gubernamentales y organizaciones no gubernamentales. Esta organización, fundada en 1948, incorpora hoy a unos 880 miembros (gubernamentales y no gubernamentales) procedentes de 133 países y es considerado como el foro más representativo de ese sector «conservacionista». Entonces, ¿cuál es el modelo que propugna?

En 1980, la UICN promovió, junto al Programa de Naciones Unidas para el Medio Ambiente y el Fondo Mundial para la Naturaleza, la llamada «Estrategia Mundial para la Conservación»(UICN-PNUMA-WWF, 1980), un documento en el que se formulaban objetivos y prioridades para la acción nacional e internacional, desde la idea de que la conservación no es la antítesis del desarrollo. Más tarde, en 1991, la misma UICN, de nuevo en forma conjunta con PNUMA y WWF, lanzó una actualización conocida coloquialmente como la «estrategia mundial de conservación para los noventa», que se publicaría bajo el nombre de «Cuidar la Tierra. Estrategia para el Futuro de la Vida» (UICN-PNUMA-WWF, 1991). Se trata, por tanto, de la propuesta más cercana a lo que podríamos entender como «el modelo conservacionista». Al leerlo, nos toparemos con que el primer tercio del documento se centra en definir los «Principios para una Sociedad Sostenible» y que todo el documento (que incluye propuestas de acciones, aplicación y seguimiento) se basa en «una estrategia mundial amplia y explícita para los cambios necesarios a fin de construir una sociedad sostenible» (p. 8 de la versión española). ¿Dónde está, pues, la diferencia que nos pide conocer la materia de bachillerato y llega a ser exigida por uno de sus criterios de evaluación?

Si partimos del Informe de la Comisión Brundtland, ya aludido, como primer hito en la definición de la idea de desarrollo sostenible, encontraremos que éste se define como aquél «que satisface las necesidades de la generación presente sin comprometer la capacidad de las generaciones futuras para satisfacer sus propias necesidades» (punto 1 del capítulo 2), y que se contrapone a modelos de desarrollo incapaces de asegurar dicha «sostenibilidad» (por tanto, «insostenibles»), que son, por otra parte, los que dominan el panorama internacional y cuyas secuelas son analizadas por el informe. Algo semejante encontraremos en el documento «Cuidar la Tierra», donde el desarrollo sostenible se define como «mejoramiento de la calidad de vida humana dentro de la capacidad de carga de los sistemas sustentadores de vida», o en la propia Agenda 21. En todos los casos, la confrontación se da entre los modelos de desarrollo sostenible (para los que, ciertamente, se han formulado muchas definiciones y requieren de una progresiva «construcción») y modelos «insostenibles» (que, además, resultan ser los mayoritariamente vigentes y domi- 
nantes en el mundo). No hay forma de encontrar la pretendida identidad de un «modelo conservacionista» contrapuesto al del «desarrollo sostenible», y tampoco lo hallaremos en la abundante literatura generada por pensadores y proponentes teóricos de las ideas del «desarrollo sostenible», como Daly, Costanza, Bartholomew, El Serafy, Gallopin... ¿Cómo puede, pues, el profesorado acometer la enseñanza de esta contraposición y de qué manera puede el alumnado enfrentarse al criterio de evaluación aludido?

No sólo la participación del sector «conservacionista» en la génesis del «desarrollo sostenible, sino incluso su carácter precursor, es algo comúnmente reconocido por quienes teorizan sobre la reciente historia de esta propuesta (Jiménez Herrero, 1996); no parece, por tanto, defendible el planteamiento - más que confuso, erróneode los contenidos de la materia de bachillerato, que, además, llega a reforzarse en los criterios de evaluación, aumentando con ello la gravedad de sus consecuencias.

\section{Los criterios de evaluación}

Hay que insistir en la importancia de esta parte del currículo oficial, que define algo así como los contenidos básicos, y que, en materias como ésta, tiene un valor particularmente destacado, por cuanto es orientación clave para la elaboración de las pruebas de acceso a la universidad, en las que el «control» por parte del profesorado que ha impartido la materia es mucho menor que en el caso de su propia evaluación durante el bachillerato.

Cabe exigir, por tanto, que los criterios de evaluación en una materia susceptible de ser examinada en la PAU sean especialmente equilibrados y coherentes. Sin embargo, en el conjunto de los once criterios de evaluación aprobados para esta materia tan compleja y amplia, se advierte una considerable dosis de heterogeneidad que poco contribuye a lo anterior. De hecho, el primer criterio («Explicar algunas repercusiones que las alteraciones medioambientales provocadas por el hombre pueden producir en la naturaleza») inicia la serie de una forma tan amplia y abstracta que casi ampara cualquier cuestión: después de ese criterio casi sobran todos los demás, piensan algunos.

Otro criterio posterior, partiendo de una idea correcta que trata de enlazar con el objetivo central de la materia en relación con los procedimientos, se revela, sin embargo, como prácticamente imposible de ser llevada a la PAU (por supuesto, cabe aceptar que pueda serlo, de una forma más equilibrada, a lo largo del curso, pero no hay que olvidar la importancia, tal vez exagerada, pero real, que sigue teniendo el examen de acceso a la universidad tanto para los alumnos de estos cursos como para la determinación de las actividades de enseñanza por parte del profesorado). Se trata del criterio 3 ( «Planificar una investigación para evaluar los riesgos más frecuentes,...»): ¿Cómo hacerlo valer en las pruebas de acceso? ¿Cómo evitar que, si no entra en aquéllos, pierda relevancia para el profesorado?
Por otra parte, se advierte un exceso de especificidad en algunos criterios (sobretodo teniendo en cuenta que sólo hay once para toda la materia) que escorarán de forma injustificada la enseñanza y el aprendizaje de los contenidos de la materia, que se quieren globales, hacia el detalle poco relevante. En este caso está el de «Utilizar técnicas químicas y biológicas para detectar el grado de contaminación presente en muestras de agua,..», cuya parte explicativa posterior hace explícita referencia a técnicas como la DBO, el oxígeno disuelto, etc. Si ya es cuestionable la elección (repito: entre tan sólo once criterios que se entiende han de establecer los «mínimos» a aprender por todos) de las técnicas bioquímicas de detección de la contaminación de aguas, la identificación de este único tipo de técnicas de detección entre los criterios de evaluación hace que éstas se conviertan en una parte «fundamental» en el trabajo educativo de la materia. ¿Pero lo son? ¿Y por qué no la medición de la erosionabilidad, o de la contaminación del aire, o de la pérdida de biodiversidad...? Numerosos profesores, además, atendiendo a la explicación de que los alumnos tendrán que saber «a partir de que ellos diagnostiquen su grado de adecuación [de las aguas] para el desarrollo de la vida o el consumo humano», terminan haciendo de las tablas y de los valores legales de calidad de aguas un objetivo central del aprendizaje, abriendo un campo que parece francamente poco justificable en una materia de estas características, globalidad y nivel educativo. Pero la PAU, puede luego pedir...

En otros criterios se advierten también excesivas concreciones en un marco, a su vez, excesivo. Es el caso de «Evaluar el impacto ambiental de un proyecto...», en donde se exige de forma «pretenciosa» para el nivel educativo una capacidad que es, en realidad, una técnica compleja de carácter preventivo, entre otras. ¿Por qué no, entonces, «ser capaz de planificar el medio físico», «ordenar el territorio» o «hacer una ecoauditoria»?... Aunque ha trascendido más entre la ciudadanía el tema de la EIA, parecería recomendable que la materia le encontrase una ubicación y orientación coherente con el tipo de técnica que representa, así como un grado de profundidad en la exigencia de su conocimiento que parezca adecuado, lo que no ocurre ahora. Además, en la explicación del criterio de evaluación, la referencia a una técnica precisa (la matriz de Leopold) termina orientando la enseñanza de las medidas preventivas (que podrían ser el contenido real de aprendizaje) hacia el uso y abuso de estas matrices que, si bien representaron un inicio histórico en las técnicas de EIA, hoy ya apenas se utilizan. ¿No es más sensato, en todo caso, conocer algo acerca de la existencia y tipo de técnicas de prevención? (y, si la EIA se considera suficientemente importante, saber del procedimiento -estudio, evaluación y declaración de impacto ambiental- más que de la aplicación de una técnica específica y, además, ya casi abandonada).

De igual modo, el sesgo dado por la especificidad de los contenidos de muchos de los criterios hace que la habitual traducción de los criterios de evaluación en pautas de orientación acerca de lo fundamental a enseñar/ aprender constituya un grave problema en la aplicación 
práctica de la materia a las aulas (y en contra de las pretensiones y objetivos, correctos y justificados, de la misma). Por plantear algunos otros ejemplos, valgan los siguientes: el estudio del problema de la contaminación atmosférica, desde los criterios de evaluación, nos llevaría a centrarnos detalladamente en la capacidad difusora de la atmósfera; el estudio de la biosfera nos remite a la mal llamada «regla del diez por ciento» en la transmisión de energía dentro de una red trófica; no aparecen cuestiones básicas respecto al tema de la incapacidad o desatención por parte del sistema socioeconómico por cerrar los ciclos de materia; la insistencia de los contenidos en el tema de los recursos se reduce, en los criterios propuestos, a la cuestión de las fuentes de energía o a la propuesta de medidas comunitarias...

En resumen, más que definir aspectos claves y básicos, y servir de orientación global sobre los contenidos y los enfoques, los criterios confunden y sesgan inadecuadamente. Eso no parece razonable en ninguna materia, pero mucho menos en una que es novedosa y susceptible de ser calificada en una prueba de acceso a la universidad, lo que debería llevar a su reconsideración.

\section{Una propuesta de ordenación de los contenidos de cara a la programación}

Llegado este punto, parece preciso trascender del análisis crítico a una propuesta de reorganización de los contenidos, tratando con ello de posibilitar una visión más coherente con lo que se ha dicho.

Hay que recordar que, desde el punto de vista que aquí se defiende, lo más adecuado resultaría la división de la materia en dos: unas ciencias de la Tierra (entendidas desde el punto de vista anteriormente expuesto) y unas ciencias ambientales. Aquí sólo se planteará una posible organización de los contenidos para esta segunda materia. De mantenerse en su actual configuración, lo menos malo nos parece diferenciar dos partes independientes dentro de la materia, siguiendo el título. Sin embargo, creemos que el tiempo disponible no es en absoluto suficiente para desarrollar mínimamente ninguna de las dos, por lo que, en realidad, sería una frustración tanto para la una como para la otra.

Para las ciencias ambientales, se parte de la aceptación de que la mayor parte de los contenidos existentes en la actual CTMA resultan válidos (a excepción de los que se refieren algo más a las ciencias de la Tierra expuestos sobre todo en «Sistemas terrestres»), aunque deberían ser organizados de diferente forma, con otro enfoque, importancia y categorización. Posteriormente, estas nuevas orientaciones deberían verse reforzadas en los criterios de evaluación.

Según esto, los contenidos ambientales podrían categorizarse conforme a la siguiente «taxonomía»:

a) Contenidos que permiten orientar la metodología o didáctica más recomendable para la materia
Se trata fundamentalmente de los que ya aparecen incluidos en el currículo vigente bajo el epígrafe «aproximación al trabajo científico». A ellos debieran añadirse aquellos métodos de enseñanza-aprendizaje que, resultando ser procedimientos propios del trabajo científico, aparecen como idóneos para esta materia, entre los que están los estudios de casos, las simulaciones, la solución de problemas, las pequeñas investigaciones...

Naturalmente, este bloque tiene un sentido fundamental de orientación didáctica y metodológica, y, aunque, lógicamente, deben comportar aprendizajes concretos de aprehensión de métodos y procedimientos de indagación, investigación, razonamiento, etc., su finalidad es convertirse en una herramienta del saber hacer que se aprende, utiliza y adquiere mediante su aplicación en el aprendizaje de otros contenidos. Se trataría, en la clasificación habitual de contenidos, de procedimientos de tipo muy general (Coll, Pozo, Sarabia y Valls, 1992).

b) Contenidos capaces de proveer de un enfoque general y un «núcleo duro» conceptual a la materia

Incluye la teoría de sistemas, entendida fundamentalmente desde su capacidad de proveer un «enfoque sistémico» fundamental para la comprensión del «medio ambiente», que constituye el verdadero «núcleo duro» conceptual. Es decir, son el objeto de estudio, comprensión e interpretación (el medio ambiente) y la «herramienta» capaz de facilitarnos ese estudio, comprensión e interpretación (el enfoque de sistemas); algo semejante a lo que ya Rosnay (1977) denominó en su momento como el macroscopio. La teoría de sistemas, por tanto, adquiere un sentido perceptivo y casi instrumental: de hábito en la forma de enfocar el estudio del medio ambiente y los problemas ambientales, huyendo, por tanto, de la tentación de ser convertida en una «lección» diferenciada y casi aislada del programa, como está ocurriendo en la actualidad en algunos casos. En este sentido, además, de las tres versiones existentes para el concepto de medio ambiente (Tabla I), la materia elegiría preferentemente la concepción sistémica.

\section{c) Contenidos que representan conceptos clave}

Son aquellos contenidos e ideas procedentes del enfoque sistémico que han de ser aplicados y utilizados en la construcción conceptual del sistema ambiental (su modelización) y en la interpretación de la dinámica y funcionamiento de los sistemas natural y socioeconómico, resultando la vía por la que se interpretan y explican los problemas ambientales dentro de un marco global. Se trata de conceptos del tipo de las entradas o inputs de los sistemas (recursos), salidas o outputs (residuos), modificaciones (impactos), alteraciones de procesos (riesgos inducidos), dinámicas (procesos) o alteraciones de ocupación y límites (capacidad de carga o acogida), etc. Sólo bajo estos esquemas sistémicos es posible dar una coherencia conceptual a una materia compleja y global, huyendo de su conversión en un anecdotario interminable de datos y situaciones (que sólo adquieren sentido «enganchados» en este tipo de esquemas). 
d) Contenidos que son métodos o técnicas de diverso tipo

Además de los procedimientos de carácter más general, que determinarán metodologías didácticas apropiadas para la adquisición de esos y otros contenidos, hay otro tipo de procedimientos específicos que constituyen métodos o técnicas concretos. Pueden ser ordenados en tres escalas de grado o nivel diferente, siendo muy importante la percepción de los tres tipos y el conocimiento de lo que representan. Dada la complejidad de unos y la variedad de otros, los objetivos educativos aquí deben ser bien ponderados, de forma que en unos casos se buscará sólo la comprensión global del sentido y aplicabilidad de tales métodos, y, en otros, se podrán seleccionar y estudiar/aplicar técnicas concretas en función de las programaciones desarrolladas, aunque bien pudieran elegirse otras distintas en casos diferentes; lo importante, por tanto, no es el dominio de una técnica específica (en las más concretas), sino conocer su existencia y sentido y, como en el estudio de casos, llegar a aplicar alguna de ellas en una situación concreta y dotada de sentido/significación.

La clasificación jerárquica en tres niveles (de más concreto a más global) podría ser la siguiente: en primer lugar, las técnicas de medición (primer nivel); en segundo lugar, los métodos o técnicas de valoración, los de toma de decisiones técnicas y los de gestión técnica (segundo nivel); y, en tercer lugar, los sistemas de ordenación territorial y planificación ambiental (tercer nivel). Sobre esta clasificación general es posible asentar el sentido y objeto de los aprendizajes que se pretenden.

Algunas de las técnicas, sobre todo del «primer nivel», son contenidos de «apoyo». Variarán, por tanto, de acuerdo con las programaciones de aula que haga cada centro. Es decir, si para tratar sobre un contenido importante de la materia, como pueden ser los problemas de contaminación, en un centro se elige trabajar en detalle sobre un tipo concreto de aquéllas, como la eutrofización para la contaminación del agua, entonces resultará necesario utilizar técnicas de medida de este tipo de contaminación acuática o con listas y normativas relativas. Pero ello no quiere decir que éstos constituyan contenidos «a aprender» para «ser exigidos» en las pruebas homologadas de acceso. Sí lo serán aquellos aspectos globales de la contaminación (de aguas, atmosférica, de suelos...) cuya comprensión como problemas ligados a la acumulación, toxicidad o capacidad de alteración de las dinámicas naturales del ecosistema revista un sentido que los hagan necesarios para comprender globalmente los problemas ambientales derivados de «no cerrar los ciclos de materia» o de «introducir sustancias nocivas, tóxicas o peligrosas» en los sistemas naturales. Pero no será ni necesario ni posible pretender que se tengan en cuenta (y menos, que se exijan) todos y cada uno de los innumerables aspectos que se podrían trabajar en la materia para comprender el fenómeno de la contaminación y que variarán en función del tipo de actividades y la elección concreta de aspectos que cada centro o equipo de profesores haya elegido en la aplicación a su ámbito de concreción curricular (su programación de aula). Exigir el aprendizaje de unos tipos concretos de técnicas muy específicas (de medición, por ejemplo) de entre las muchas existentes para distintos fenómenos o procesos ambientales sólo nos llevará a restringir el campo de aplicación concreta de la materia en cada centro y al empobrecimiento y la rigidez de una materia que precisa de una gran diversidad de tratamientos posibles en vistas a su complejidad, dada su capacidad de adaptación amplia a distintos lugares, intereses y formas de desarrollarla. Por ejemplo, puede parecer importante conocer algo sobre técnicas de medición de contaminación atmosférica en un centro inmerso en un

Tabla I

Tipos de concepciones acerca del medio ambiente.

\begin{tabular}{|l|l|}
\hline TIPOS & EJEMPLOS \\
\hline $\begin{array}{l}\text { De carácter amplio e indefinido, } \\
\text { enumerativas... }\end{array}$ & $\begin{array}{l}\text { Marco o entorno vital } \\
\text { Conjunto de agentes físicos, químicos, biológicos y de los factores sociales susceptibles de } \\
\text { causar un efecto directo o indirecto, inmediato o a plazos, sobre los seres vivos y las actividades } \\
\text { humanas }\end{array}$ \\
$\begin{array}{l}\text { Dotadas de un carácter parcial } \\
\text { o relativo a un punto de referencia } \\
\text { Conjunto de fuerzas o condiciones que actúan sobre un organismo, población o comunidad } \\
\text { (O'Riordan, 1971) } \\
\text { Entorno que influye o condiciona un organismo o conjunto de organismos } \\
\text { Sistema complejo de interrelaciones inertes, vivas y socioculturales, que nos proporciona } \\
\text { una nueva dimensión científica bajo el concepto de ciencia del ambiente o ciencia ambiental } \\
\text { (Jiménez Herrero, 1989) } \\
\text { Sistemas multidimensionales de interrelaciones complejas en continuo estado de cambio }\end{array}$ \\
\hline
\end{tabular}


ambiente urbano-industrial como Madrid, pero tal vez resulte más interesante saber acerca de la existencia de métodos de medición de la erosión o de la pérdida de suelos en áreas con problemas de desertificación, como Murcia; o sobre contaminación orgánica de aguas en una localidad aguas abajo de una zona de industrias o ciudades con efluentes orgánicos importantes.

El objetivo educativo en todos los casos deberá ser «conocer de la existencia de métodos y técnicas de medición capaces de proveer de información objetiva sobre las características y grado de contaminación o de alteración ambiental», incluso «haber manejado o aplicado alguna de las técnicas». Pero probablemente ninguno de los dos casos tenga por qué convertirse en uno de los once criterios de evaluación básicos para la materia, ya que eso nos llevaría en la práctica a excluir el tratamiento de los restantes en vista de la preponderancia exagerada y arbitraria dada a uno de ellos por el currículo oficial.

e) Contenidos centrales del trabajo educativo: los problemas ambientales

Se trata del aspecto central que, a nuestro juicio, debiera organizar la mayor parte de las programaciones y, por tanto, de las actividades de enseñanza-aprendizaje. Los problemas ambientales pueden ser seleccionados $u$ organizados mediante diferentes criterios, como son su ubicación en la escala local-global, su mayor relación con un tipo u otro de causalidad central (ligados al uso de los recursos, ligados a la ocupación o alteración de sistemas naturales o ligados a la producción de residuos...), su mayor o menor complejidad causal, etc. Lo importante, en este caso, es que se aborde el estudio y comprensión de los principales tipos, de forma que se permita alcanzar no sólo la comprensión de éstos, sino, además, que se haya podido desarrollar un buen conjunto de herramientas conceptuales y procedimientos de análisis e interpretación de diferentes tipos de problemáticas ambientales, consiguiendo una visión suficientemente global e interactiva (sistémica) de la crisis ambiental.

f) Contenidos que constituyen objetivos finales de carácter ético y social

La comprensión e interpretación racional de los problemas ambientales en una forma global y sistémica no puede constituir el último paso ni en los contenidos ni en los objetivos de la materia; falta buscar el aprendizaje sobre la «construcción» de alternativas viables, éticas y razonadas capaces de enfrentarse de forma global con las causas de los problemas ambientales que han sido científica y socioeconómicamente analizados (y no sólo conocer algo acerca de las técnicas o métodos de medición o de reducción de impactos concretos). Este contenido fundamental trataría, pues, de todo lo relacionado con la generación de alternativas y soluciones globales a las causas de los problemas ambientales, es decir, la construcción de modelos de sostenibilidad para las actividades humanas, la consideración de la solidaridad inter e intrageneracional como objetivo ético y ambien- tal, la integración de desarrollo y conservación, etc.; en definitiva: la construcción de una sociedad mundial sostenible e integrada en su medio ambiente. Este contenido, que representará siempre un objetivo final irrenunciable para las ciencias ambientales, puede ir siendo construido parcialmente según se van desarrollando las capacidades de interpretación de lo que ocurre y su porqué en cada problema ambiental trabajado; probablemente, algunas de estas pautas puedan ir siendo motivo de enseñanza desde los primeros momentos de la materia, pero, desde luego, habrán de constituir un contenido recapitulador final en la programación.

Desde esta «taxonomía» de contenidos puede asignarse un «papel» específico a cada tipo, lo que permitiría dar coherencia y «homogeneidad» a las diferentes formas de desarrollar la materia en los distintos centros, sin renunciar por ello a la complejidad inherente de las ciencias ambientales y sin caer en el reduccionismo y el sesgo que hacen peligrar la visión necesariamente sistémica de la aproximación ambiental.

\section{Sobre la formación ambiental limitada a los bachille- ratos científicos}

Curiosamente, la polémica acerca de la ubicación de la CTMA no parece haber trascendido a un ámbito más importante del que en su momento caldeó los ánimos en varios foros. Me refiero a la limitación de la materia, en su calidad de «materia de modalidad» a uno sólo de los bachilleratos (y ni eso, puesto que, en realidad, es materia de modalidad de uno sólo de los dos tipos en que ésta se subdivide). Rescatando la idea de unas ciencias ambientales capaces de favorecer el aprendizaje de los factores complejos que explican y recrean los problemas ambientales y el debate riguroso, científico y social, acerca de las vías para su solución, ¿qué justifica que sólo aquellos alumnos y alumnas que hayan optado por las ciencias de la naturaleza y la salud la cursen? ¿No resulta francamente útil esta aproximación científica en la formación de los bachilleres en tecnología? ¿Acaso un futuro estudiante de ingeniería no debiera haber cursado esta materia y sus contenidos en su bachillerato? ¿Qué decir de los estudiantes de económicas o de sociología, que habrán transitado por el bachillerato de humanidades? (A estas alturas de siglo y de problemática ambiental, resulta casi impertinente la reiteración de estas preguntas.)

Es difícil justificar la ausencia de troncalidad de una materia específica de ciencias ambientales en todas estas modalidades. No lo es, probablemente, el estudio de las ciencias de la Tierra, que sí debieran encontrar un espacio propio en el bachillerato científico. En ese sentido, sólo caben esperar beneficios de la separación de ambos títulos en materias diferentes. Por otra parte, tal vez sí sea positiva la diversificación de la materia entre distintos departamentos didácticos según las diferentes modalidades de bachillerato: ello permitiría no sólo un reparto de horas entre profesorado de distintas especialidades, sino también un sesgo más próximo al sentido general de la modalidad, aunque sobre unos planteamientos curri- 
culares comunes. Esa generalización de la formación de los bachilleres en ciencias ambientales permitiría recoger un objetivo muy reiterado en diversos ámbitos, así como diversificar las necesidades de actualización del profesorado en cuestiones ambientales y multiplicar los enfoques y, consecuentemente, la generación de materiales, recursos y elementos didácticos en un sentido que parece incuestionable para las necesidades educativas del inminente siglo XXI.

\section{COLOFÓN}

No querría terminar sin insistir, aun a fuerza de parecer reiterativo, en que la materia de «ciencias ambientales» es no sólo necesaria, sino fundamental en los nuevos

\section{REFERENCIAS BIBLIOGRÁFICAS}

AEPECT. (1992). Una nueva materia en el bachillerato de ciencias. Enseñanza de las Ciencias de la Tierra, 0, pp. 45.

ANGUITA, F. (1994). Geología, ciencias de la tierra, ciencias de la naturaleza: paisaje de un aprendizaje global. Enseñanza de las Ciencias, 12(1), pp. 15-21.

ANGUITA, F. (1996). La evolución de la tectónica de placas: el «nuevo» interior de la Tierra. Enseñanza de las Ciencias de la Tierra, 3(3), pp. 137-148.

BOLÓS, M. (1992). Manual de ciencia del paisaje. Barcelona: Masson, SA.

COLL, C., POZO, J.I., SARABIA, B. y VALLS, E. (1992). Los contenidos en la reforma. Enseñanza y aprendizaje de conceptos, procedimientos y actitudes. Madrid: Aula XXISantillana.

GONZÁLEZ BERNÁLDEZ, F. (1981). Ecología y paisaje. Madrid: H. Blume Ediciones.

JIMÉNEZ HERRERO, L. (1989). Medio ambiente y desarrollo alternativo. Madrid: IEPALA Editorial.

JIMÉNEZ HERRERO, L. (1996). Desarrollo sostenible y economía ecológica. Madrid: Síntesis.

KING, C. (1992). Las ciencias de la Tierra en el currículo nacional del Reino Unido: pasado, presente y futuro. Madrid: CIDE-MEC. (No editado)

KUHN, T. (1962). The Structure of Scientific Revolutions. Chicago: University of Chicago Press. Trad. cast., 1971, La estructura de las revoluciones científicas. México: Fondo de Cultura Económica.

MARGULIS, L. y OLENDZENSKI, L. (eds.). (1992). Environmental Evolution. Effects of the Origin and Evolution of Life on Planet Earth. Massachusetts: Massachusetts Institute of Technology. Trad. cast., 1996, Evolución Ambiental. bachilleratos (en todos), al igual que parece más que razonable que las «ciencias de la Tierra» encuentren, en su perspectiva más global, un hueco adecuado dentro del bachillerato de ciencias. Así, aunque el análisis y las reflexiones anteriores puedan parecer muy críticas (y lo sean), parten del reconocimiento y respeto por el trabajo de quienes han tenido que acometer en su momento la tarea de elaborar el ahora vigente currículo. Sin duda, coincidimos en que es más fácil criticar y descubrir fallos sobre lo ya hecho que iniciar la construcción de algo desde prácticamente nada. Sin embargo, también creemos que cada momento exige una tarea y ahora toca la de reformar, actualizar y modificar lo existente que sea problemático, en un camino de renovación constante. Pienso que las características (muy particulares) de la materia, más que pedirlo, empiezan a exigirlo. Por eso, este texto parte de la esperanza de contribuir a abrir un debate que nos conduzca un nuevo paso más adelante.

Efectos del origen y evolución de la vida sobre el planeta Tierra. Madrid: Alianza Editorial.

MILLER, G.T. (1992). Living in Environment. An Introduction to Environmental Science. EUA: Wadsworth Publishing Co. Trad. cast., 1994, Ecología y medio ambiente. Grupo Editorial Iberoamérica.

O'RIORDAN, T. (1971). Environment management. Progress in Geography, 3, pp. 173-231

ROSNAY, J. (1977). El macroscopio. Madrid: AC.

SÁEZ, M.J. y RIQUARTS, K. (1996). El desarrollo sostenible y el futuro de la enseñanza de las ciencias. Enseñanza de las Ciencias, 14(2), pp. 175-182.

TRICART, J. y KILLIAN, J. (1972). L’Èco-géographie et l'aménagement du milieu naturel. París: Librairie François Maspero. Trad. cast., 1982, La ecogeografía y la ordenación del medio natural. Editorial Anagrama.

UICN-PNUMA-WWF. (1980). Estrategia mundial para la conservación. Gland: UICN.

UICN-PNUMA-WWF. (1991). Cuidar la Tierra. Estrategia para el futuro de la vida. Gland: UICN.

WILSON, J.T. (1968). Revolution dans les Sciences de la Terre. Vie et Milieu, XIX, 2B: 395-424 (En el número 1(2) de 1993 de Enseñanza de las Ciencias de la Tierra (pp. 7285) se encuentra reproducido este artículo en un homenaje de esta revista al prestigioso investigador de la universidad de Toronto).

WORLD COMMISION ON ENVIRONMENT AND DEVELOPMENT. (1987). Our Common Future. Oxford: Oxford University Press.Trad. cast., 1988, Nuestro Futuro Común. Madrid: Alianza Editorial. 